Research Paper

\title{
Long non-coding RNA MAGI2-AS3 inactivates STAT3 pathway to inhibit prostate cancer cell proliferation via acting as a microRNA-424-5p sponge
}

\author{
Xin Wei ${ }^{1}$, Yi Hou ${ }^{1}$, Yan Zhang ${ }^{2}$, Huaiwei Zhang ${ }^{1}$, Zhou Sun${ }^{1}$, Xiangdi Meng${ }^{1}$, Zhixin Wang1 ${ }^{\bowtie}$ \\ 1. Department of Urology, China-Japan Union Hospital of Jilin University, 126 Xiantai Street Changchun 130033, P.R.China \\ 2. Department of Endocrinology, China-Japan Union Hospital of Jilin University, 126 Xiantai Street Changchun 130033, P.R.China \\ $\square$ Corresponding author: Zhixin Wang, Department of Urology, China-Japan Union Hospital of Jilin University, 126 Xiantai Street Changchun 130033, \\ P.R.China. E-mail: wang_zx@jlu.edu.cn. \\ (c) The author(s). This is an open access article distributed under the terms of the Creative Commons Attribution License (https://creativecommons.org/licenses/by/4.0/). \\ See http://ivyspring.com/terms for full terms and conditions.
}

Received: 2021.03.22; Accepted: 2021.09.21; Published: 2022.01.01

\begin{abstract}
Aberrant expression of long non-coding RNAs (IncRNAs) that results in sustained activation of cell growth promoting pathways is an important mechanism in driving prostate cancer progression. In the present study, we explored differentially expressed IncRNAs in two microarray datasets of prostate benign and malignant tissues. We found that MAGI2-AS3 was one of the most downregulated IncRNAs in prostate tumors, which was further confirmed in our collected clinical samples. The function assays showed that MAGI2-AS3 overexpression decreased cell viability and led to obvious cell apoptosis in PC-3 and DU145 prostate cancer cells. Elevation of MAGI2-AS3 decreased the activity of STAT3 in PC-3 and DU145. In addition, microRNA-424-5p (miR-424-5p), a positive regulator of STAT3 pathway, was predicted as a target of MAGI2-AS3, furthermore, the interaction between MAGI2-AS3 and miR-424-5p was confirmed via reverse-transcript polymerase chain reaction (RT-qPCR), dual luciferase reporter assay and RNA immunoprecipitation (RIP). MAGI2-AS3 upregulated miR-424-5p and downregulated COPI in PC-3 and DU145. More importantly, IL6-induced activation of STAT3 pathway could attenuate the biological effect of MAGI2-AS3 in PC-3 and DU145. In clinical samples, MAGI2-AS3 levels were negatively correlated with miR-424-5p expression, while positively correlated with COPI mRNA expression. Altogether, the current study revealed MAGI2-AS3 as a novel negative regulator of prostate cancer development.
\end{abstract}

Key words: MAGI2-AS3; prostate cancer; miR-424-5p; STAT3

\section{Introduction}

Prostate cancer is the most prevalent cancer type for males worldwide, accounting for nearly $19 \%$ of newly diagnosed cancer cases in United States, 2018 [1]. In China, statistics suggest that the morbidity and mortality of prostate cancer has been gradually increased in recent years [2]. As the primary treatment approach for patients with prostate cancer, androgen deprivation therapy has greatly improved the overall survival of the patients. However, acquired and de novo resistance to the inhibition of androgen receptor signaling occur in these patients, which is closely associated with the death of patients with prostate cancer [3]. Therefore, it is important to study the molecular mechanisms of prostate cancer progression and provides novel targets and biomarkers for the treatment of patients.

Long non-coding RNAs (lncRNAs) are defined as single-stranded transcripts (more than 200 nucleotides) with no protein coding potential. Previously known as "junk" RNAs, recent studies have revealed that they play central roles in mediating normal physiology processes and the development of human diseases. Mechanistically, lncRNAs can act as microRNA (miRNA) sponge, directly interact with miRNAs, leading to downregulation of miRNAs and upregulation of miRNA targets. Most recent reports 
have identified several oncogenic or tumor suppressor lncRNAs in prostate cancer via the miRNA sponge method [4-6]. For example, UCA1 (urothelial carcinoma associated 1) promotes prostate cancer progression via binding to miR-143 and increase MYO6 expression [4]. MEG3 enhances expression of QKI-5 via interaction with miR-9-5p in prostate cancer cells, therefore triggering cell apoptosis and inhibiting cell proliferation and motility [5]. The expression of MAGI2-AS3, which locates on chromosome 7q21.11, is associated with several cancer types such as breast cancer and bladder cancer [7-9]. A recent bioinformatic analysis shows that, MAGI2-AS3 in combination with 2 lncRNAs and 6 mRNAs, are signatures of prognostic prediction of patients with prostate cancer [10]. The precise molecular mechanisms and functions of MAGI2-AS3 have not been studied in prostate cancer yet.

In current study, we discovered that MAGI2-AS3 is a significantly downregulated lncRNA in prostate cancer according to previously published datasets, which was further confirmed in our collected clinical samples. In prostate cancer cell lines, we showed that $M A G I 2-A S 3$ inhibited cell proliferation and promoted cell apoptosis. In addition, MAGI2-AS3 sponged miR-424-5p, a miRNA with oncogenic potential, and inactivated STAT signaling in the studied cells. The data collectively demonstrated a tumor suppressor role of MAGI2-AS3 in prostate cancer.

\section{Materials and methods}

\section{Collection of patient samples}

109 pairs of prostate tumors and adjacent non-tumor tissues were collected during surgery in China-Japan Union Hospital of Jilin University from July 2016 to June 2019. Inclusion criteria: Patients diagnosed with prostate cancer and has provided informed consents. Exclusion criteria: Patients received chemotherapy or other therapy before the surgery. These patients were aged from 45 to 82 with the average age of 66 . The protocol of the current study was reviewed and approved by the Ethical Committee of China-Japan Union Hospital of Jilin University. These tissues were stored in $-80^{\circ} \mathrm{C}$ before subjected to RNA extraction.

\section{Cell culture and treatment}

The immortalized prostate myofibroblast stromal cell line WPMY-1, prostate cancer cell lines PC-3 and DU145 were obtained from ATCC (Manassas, VA). These cells were maintained in DMEM (Gibco; Thermo Fisher Scientific) supplemented with $10 \%$ FBS (Hyclone; Thermo Fisher Scientific). The culture condition was $5 \% \mathrm{CO}_{2}$ and $37^{\circ} \mathrm{C}$. Cells were treated with a mixture of IL6 (Sigma
Aldrich) (10 ng/mL) or equal amount of vehicle (sterilized water) and the culture medium for $24 \mathrm{~h}$. MAGI2-AS3 (Gene ID: 100505881) was inserted into pcDNA3.1 for overexpression. miR-424-5p mimic and miR-NC mimic were purchased from GenePharma (Suzhou, China). Plasmids and miRNA mimic were transfected into cells by Lipofectamine 3000 reagent (Invitrogen; Thermo Fisher Scientific).

\section{RNA extraction and realtime PCR (RT-qPCR)}

Nuclear and cytoplasm RNA was isolated by Cytoplasmic \& Nuclear RNA Purification Kit (Norgen Biotek, Ontario, Canada) following manufacturer's protocol. Tissues and cells were both treated with TRIzol reagent (Invitrogen; Thermo Fisher Scientific, Carlsbad, CA) for the extraction of total RNA. RNA was reverse transcribed into cDNA via M-MLV Reverse Transcriptase (Invitrogen; Thermo Fisher Scientific). The RT-qPCR was carried out using Taq Pro Universal SYBR qPCR Master Mix (Vazyme, Nanjing, China). The protocol was as following: step 1: $95^{\circ} \mathrm{C}$, $30 \mathrm{sec}$; step 2 : $95^{\circ} \mathrm{C}, 5 \mathrm{sec} ; 60^{\circ} \mathrm{C}, 30 \mathrm{sec}, 40$ cycles. The primers were: Bcl2-F: 5'-GGTGGGGT CATGTGTGTGG-3'; Bcl2-R: 5'-CGGTTCAGGTACT CAGTCATCC-3'; Mcl1-F: 5'-TGCTTCGGAAACTG GACATCA-3'; Mcl1-R: 5'-TAGCCACAAAGGCACC AAAAG-3'; MAGI2-AS3-F: 5'-TGGGTCTGTGCA GAGTTGAG-3'; MAGI2-AS3-R: 5'-AGGGAGTCTAG GCCCCTTCT-3'; miR-424-5p-F: 5'-GCCGAGCAGC AGCAATTCAT-3'; miR-424-5p-R: 5'-CTCAACTGGT GTCGTGGA-3'; COP1-F: 5'-TGAGTGGCTTATACTC TCCTGTC-3'; COP1-R: 5'-AACGTGCTATTATAC CAAGGCTG-3'; GAPDH-F: 5'-GGAGCGAGATCCC TCCAAAAT-3'; GAPDH-R: 5'-GGCTGTTGTCAT ACTTCTCATGG-3'; U6-F: 5'-CTCGCTTCGGCAGC ACA-3'; U6-R: 5'-AACGCTTCACGAATTTGCGT-3'. We selected GAPDH and U6 as internal controls of mRNA and miRNA, respectively. The relative expression of each gene was calculated using the comparative $\Delta \Delta \mathrm{Ct}$ method.

\section{Protein extraction and western blotting}

RIPA lysis buffer (Thermo Fisher Scientific) was selected for extraction of protein from cells. BCA kit was used to determine the protein concentration. 15 $\mu \mathrm{g}$ proteins were loaded on the SDS-PAGE gel, prior to being transferring to PVDF membrane. After blocking in 5\% non-fat milk, membrane was incubated with the primary antibody and secondary antibody sequentially. The blots were developed with ECL Western Blotting Substrate (Pierce; Thermo Fisher Scientific). The antibodies were listed as follow: STAT3 (Cat.12640, 1:2000), p-STAT3 (Tyr707) (Cat.9145, 1:1000) and GAPDH (Cat.97166, 1:10000) antibodies were obtained from CST (Danvers, MA). 
COP1 (Cat.GTX34159, 1:2000) antibody was purchased from GeneTex (San Antonio, TX). HRP-conjugated goat anti-mouse (ab6789, 1:100000) and HRP-conjugated goat anti-rabbit (ab6721, 1:100000) were products of Abcam (Cambridge, UK).

\section{Bioinformatic analysis}

The expression of MAGI2-AS3 in benign prostate $(n=14)$ and prostate cancer $(n=36)$ was downloaded from GEO database (GSE46602). The expression of MAGI2-AS3 in benign prostate $(\mathrm{n}=8)$ and prostate cancer $(\mathrm{n}=13)$ were downloaded from GEO database (GSE55945). The expression of MAGI2-AS3 in 52 normal prostate tissues and 492 prostate tumors was retrieved from GTEx and TCGA-PRAD projects. The potential target miRNA of MAGI2-AS3 was predicted by the miRDB software (http://mirdb.org/). Correlation between MAGI2-AS3 with COP1 and STAT target genes was analyzed on the GEPIA software (http:/ / gepia.cancer-pku.cn/).

\section{RNA immunoprecipitation (RIP)}

The RIP assay was applied to study the interaction between MAGI2-AS3 and miR-424-5p using the EZMagna RIP RNA-binding protein immunoprecipitation kit (Millipore) according to the manufacturer's instructions. Briefly, prostate cancer cells were lysed with RNA lysis buffer supplemented with protease and RNase inhibitors. The lysate was incubated with magnetic beads pre-coated with Ago2 antibody (Cat. 10686-1-AP, ProteinTech) or a control IgG (Cat. ab133470, Abcam) at $4^{\circ} \mathrm{C}$ overnight. Then, the RNAs were subjected to RT-qPCR to detect MAGI2-AS3 and miR-424-5p expression.

\section{Determination of cell proliferation and cell apoptosis}

The cell proliferation was assessed by the CCK-8 kit (AbMole, Houston, TX). Medium was mixed with CCK-8 solution, incubated with cells for 2 hours, then transferred to a new 96-well plate. OD 450 of each well was read by the Microplate Reader to manifest the cell number.

The percentage of apoptotic cells was detected by Annexin V-FITC/PI Apoptosis Detection Kit (Vazyme, Nanjing, China). Cells were collected and stained with Annexin V-FITC and PI sequentially. After that, cells were subjected to flow cytometry analysis and the data were analyzed with FlowJo software. Cells were distributed in 4 phases according to the PI and FITC status. FITC+/PI+ and FITC+/PIcells were the apoptotic cells.

\section{Dual luciferase reporter assay}

Full length of MAIG2-AS3 (MAGI2-AS3-WT) or the mutant MAGI2-AS3 (MAGI2-AS3-Mut) was inserted into pmir-GLO plasmid. Cells were co-transfected with pmir-GLO-MAGI2-AS3-WT or pmir-GLO-MAGI2-AS3-Mut and miR-424-5p mimic or miR-NC mimic by Lipofectamine 3000 (Invitrogen; Thermo Fisher Scientific). After 48 hours, the relative luciferase of each group was detected by the Dual-Glo Luciferase Assay System (Promega, Madison, WI). pGMSTAT3-Luc (Yeason Biotech, Shanghai, China) containing 4 binding sites for STAT3 was transfected into cells. At 24 hours after treatment, the luciferase activity was detected by the Dual-Glo Luciferase Assay System to reflect STAT3 activity.

\section{Statistical analysis}

All data were calculated and the graphs were generated by the GraphPad Prism 6.0. Student's t test was used to compare the differences between 2 groups. For the differences among 3 groups, they were analyzed by one-way ANOVA followed by Tukey test. Pearson analysis was selected to study the association between gene expression. All experiments were repeated three times. P value less than 0.05 was considered as statistically significant.

\section{Results}

\section{MAGI2-AS3 was a downregulated IncRNA in prostate cancer}

To explore the differentially expressed lncRNAs in prostate cancer, we firstly retrieved expression profiles of prostate cancer and benign prostate from GSE46602 and GSE55945. MAGI2-AS3 was found to be one of the most significantly downregulated lncRNA in prostate cancer from both GSE46602 and GSE55945 (Fig. 1A-B). For validation, we also analyzed MAGI2-AS3 expression in TCGA-PRAD dataset. Consistently, compared with 52 normal prostate tissues, MAGI2-AS3 was significantly decreased in 492 prostate cancers (Fig. 1C). We further collected patient samples. According to RT-qPCR results, MAGI2-AS3 was approximately 3-fold lower in prostate cancer than in the adjacent normal tissues (Fig. 1D). Decreased MAGI2-AS3 expression was associated with high Gleason score (Fig. 1E). MAGI2-AS3 expression was not associated with age or tumor stage (Table 1 ).

\section{Overexpression of MAGI2-AS3 inhibited prostate cancer cell proliferation}

We next detected MAGI2-AS3 expression in two prostate cancer cell lines (PC-3 and DU145) and the immortalized prostate myofibroblast stromal cell line WPMY-1. MAGI2-AS3 was decreased in PC-3 and DU145 compared with WPMY-1 (Fig. 2A). We cloned full length of MAGI2-AS3 into pcDNA3.1 plasmid. After transfection, it was found that MAGI2-AS3 
expression was increased 9-fold and 15-fold in PC-3 and DU145 cells respectively (Fig. 2B). Via separating nuclear and cytoplasm fractions of cells, we found that MAGI2-AS3 was mainly localized in cytoplasm of PC-3 and DU145 cells (Fig. 2C). Function assays showed that elevation of MAGI2-AS3 inhibited cell proliferation of both PC-3 and DU145 cells (Fig. 2D-E). In addition, we detected the percentage of apoptotic PC-3 and DU145 cells by flow cytometry analysis. We found that MAGI2-AS3 overexpression led to significant cell apoptosis in PC-3 and DU145 cells (Fig. 2F-G).

A

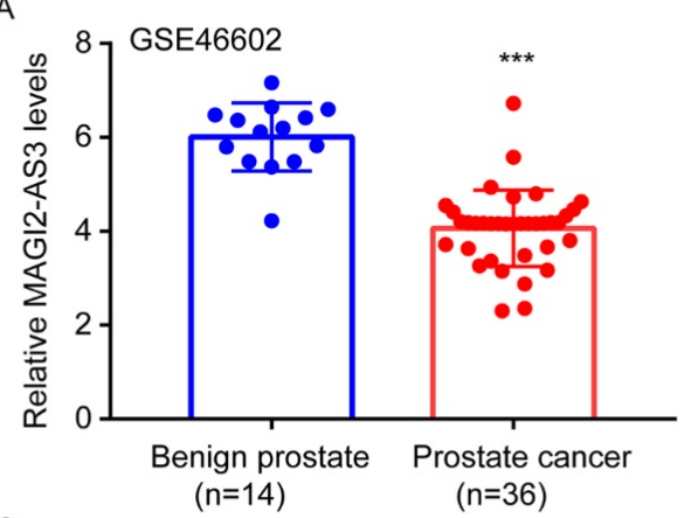

C
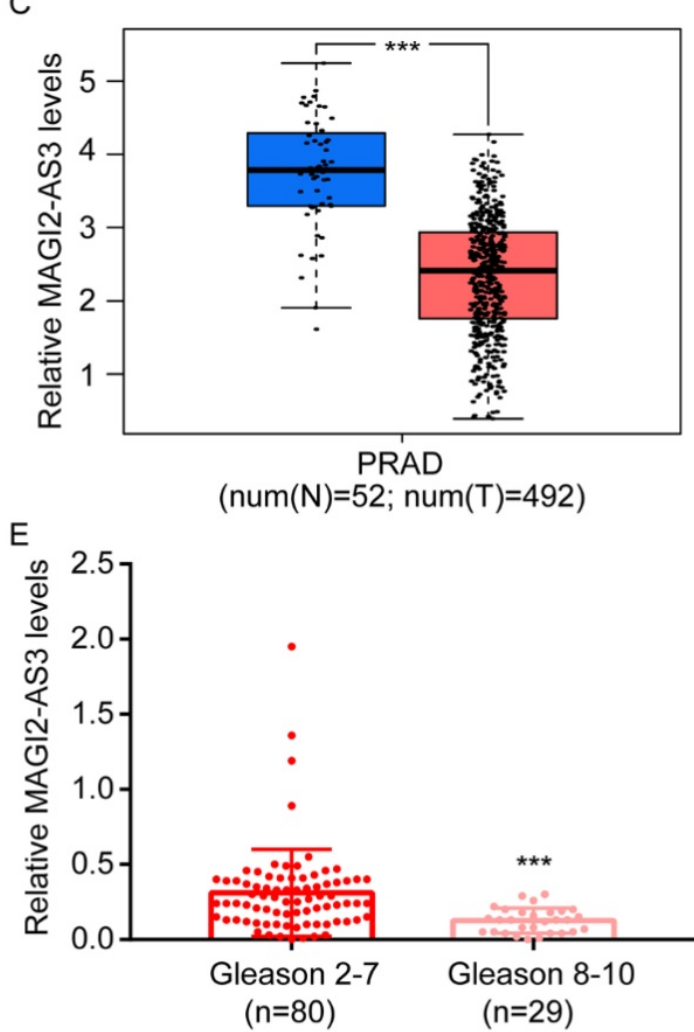

Table 1. Association between MAGI2-AS3 levels and the clinicopathological features of patients with prostate cancer

\begin{tabular}{llll}
\hline Characteristics & \multicolumn{2}{l}{ Number of cases } & p value \\
\hline MAGI2-AS3 expression & \multicolumn{2}{l}{ High } & Low \\
& 55 & 54 & \\
Age (years) & & & 0.435 \\
$\leq 60$ & 19 & 23 & \\
$>60$ & 36 & 31 & \\
Gleason score & & & 0.005 \\
$2-7$ & 47 & 33 & \\
$8-10$ & 8 & 21 & \\
Tumor stage & & & 0.441 \\
T2 & 34 & 29 & \\
T3-4 & 21 & 25 & \\
\hline
\end{tabular}

B

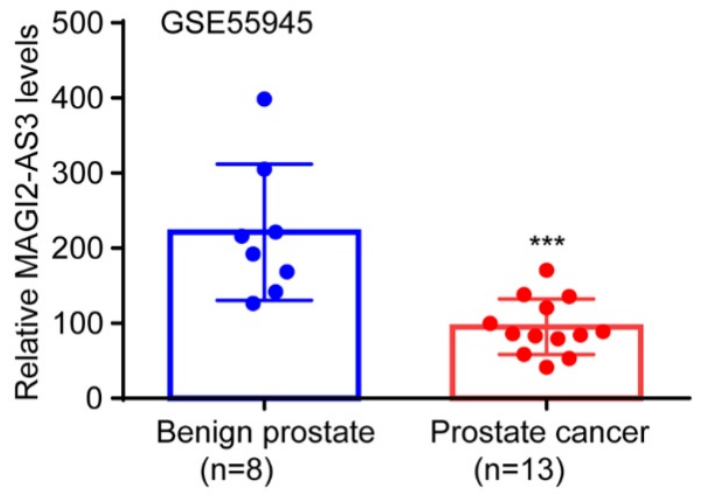

D

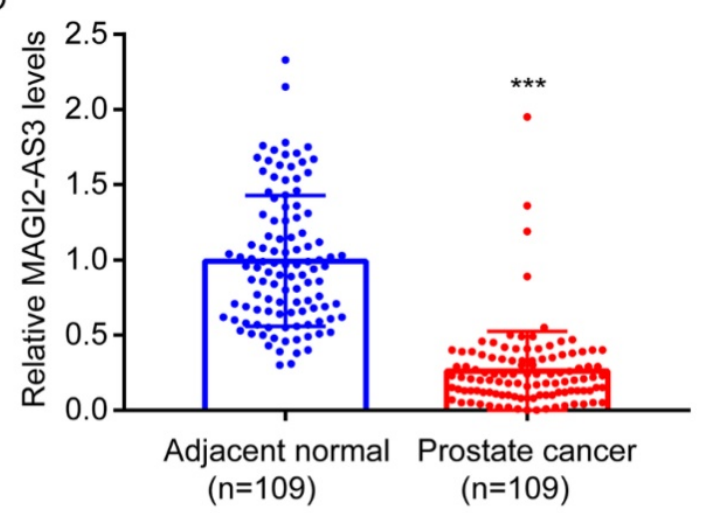

Figure 1. MAGI2-AS3 was a downregulated IncRNA in prostate cancer. A-B. Bioinformatic analysis of MAGI2-AS3 expression in benign prostate and prostate cancer tissues from GSE46602 and GSE55945 datasets. C. Bioinformatic analysis of MAGI2-AS3 expression in normal prostate and prostate cancer from TCGA dataset. D. RT-qPCR detection of MAGI2-AS3 expression in 109 pairs of prostate cancer and adjacent normal prostate. E. Comparison of MAGI2-AS3 expression between prostate tumors of high Gleason score and those of low Gleason score. $* * *, p<0.001$. 
A

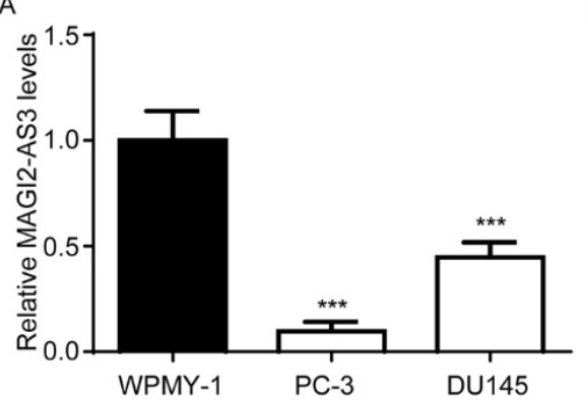

C

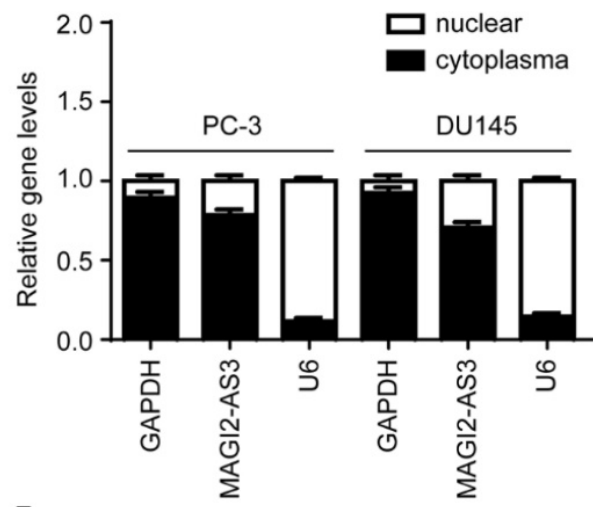

E

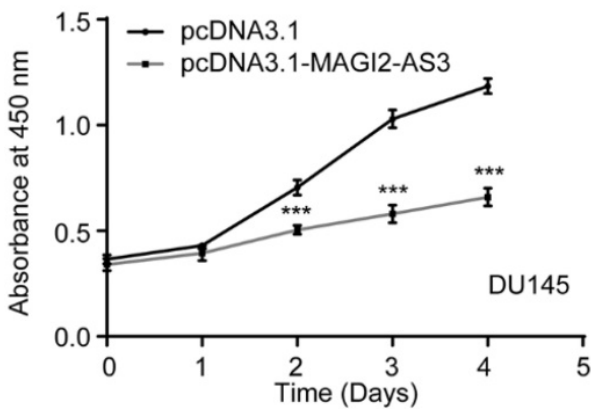

G

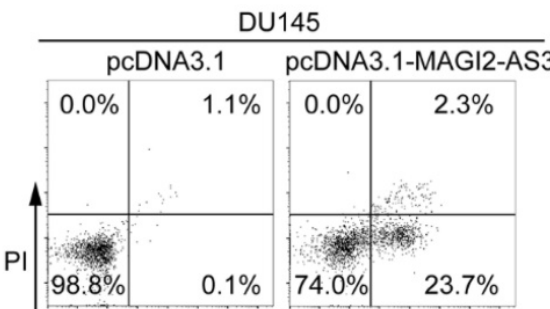

FITC

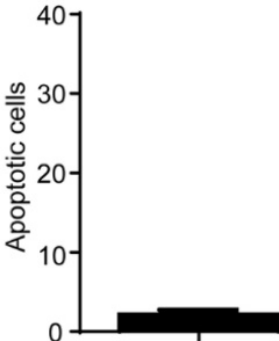

B

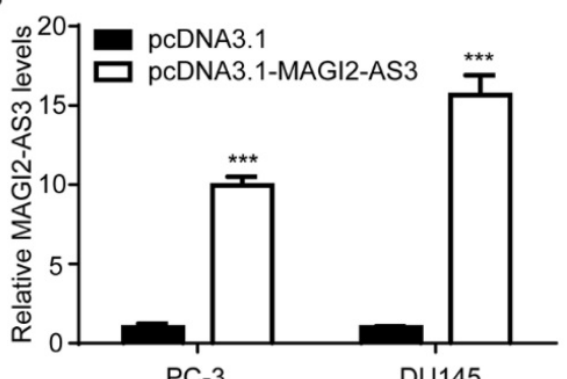

D

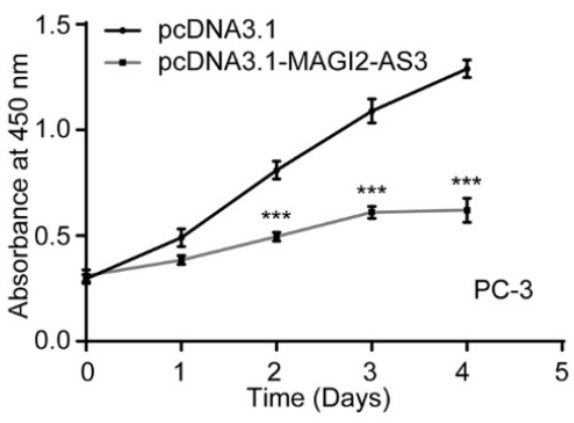

$\mathrm{F}$
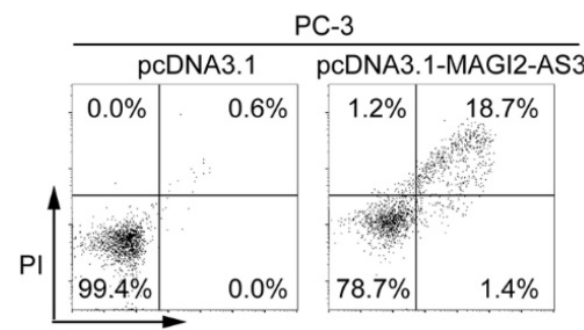

FITC

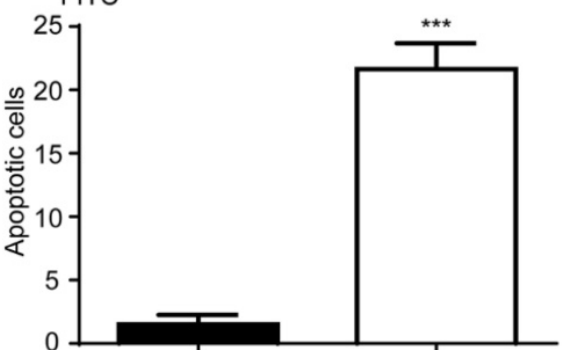

Figure 2. Elevation of MAGI2-AS3 inhibited cell proliferation and promoted cell apoptosis in prostate cancer cells. A. MAGI2-AS3 expression was detected in WPMY-1 and two prostate cancer cell lines (PC-3 and DU145) by RT-qPCR. B. MAGI2-AS3 expression was detected in PC-3 and DU145 with transfection of pCDNA3.1 or pcDNA3.1-MAGI2-AS3 by RT-qPCR. C. RT-qPCR was used to detect GAPDH mRNA, MAGI2-AS3 and U6 expression in nuclear and cytoplasm fractions of PC-3 and DU145 cells. $\mathrm{D}$-E. The cell proliferation ability was detected in PC-3 (D) and DU145 (E) cells with transfection of pcDNA3.1 or PcDNA3.1-MAGI2-AS3. F-G. The percentage of apoptotic cells was detected in PC-3 (F) and DU145 (G) cells with transfection of pcDNA3.1 or pcDNA3.1-MAG12-AS3. ***, p<0.001. 
A

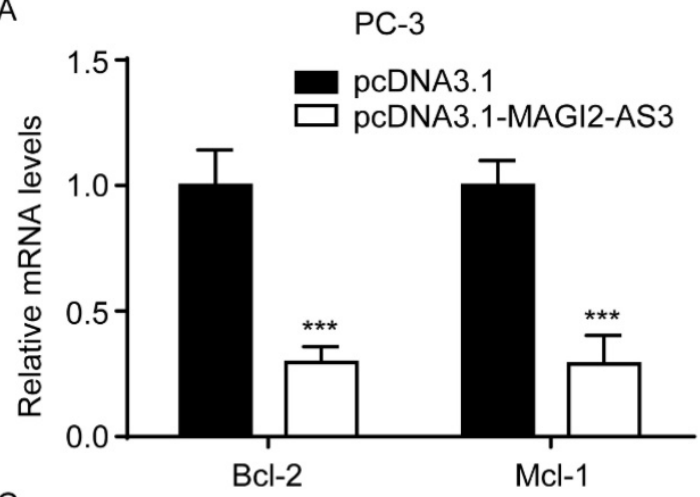

C

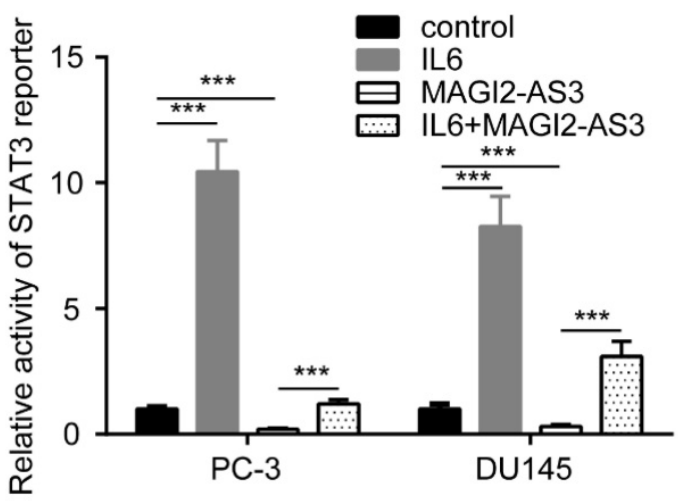

B

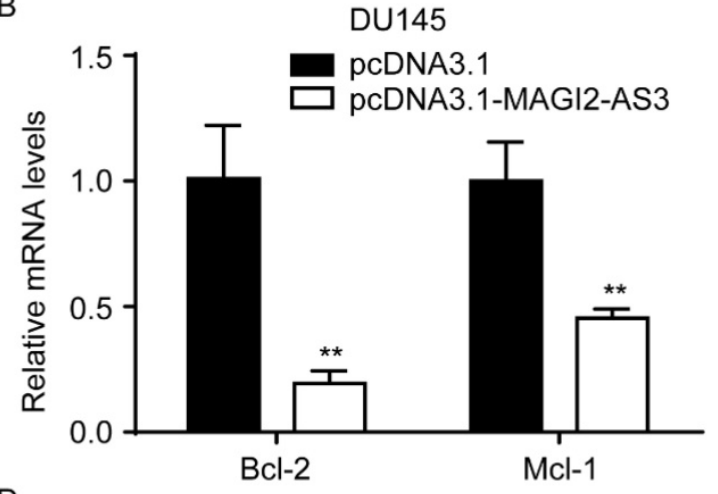

D

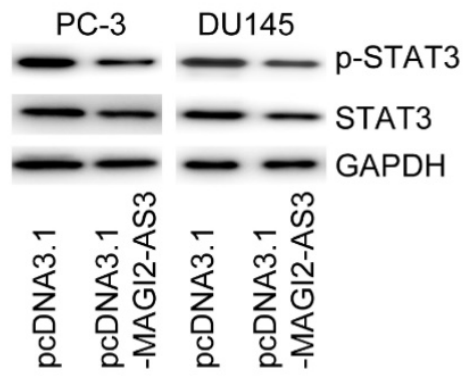

Figure 3. MAGI2-AS3 inactivated STAT3 signaling in prostate cancer cells. A-B. Bcl-2 and Mcl-1 mRNA levels were determined in PC-3 (A) and DU145 (B) cells with transfection of pcDNA3.1 or pcDNA3.1-MAGI2-AS3. C. STAT3 reporter activity was detected in PC-3 and DU145 cells with transfection of pcDNA3.1 or pcDNA3.1-MAGI2-AS3 and treatment of IL6. D. Total STAT3 and p-STAT3 protein levels were measured in PC-3 and DU145 cells with transfection of pcDNA3.1 or pcDNA3.1-MAGI2-AS3. **, $\mathrm{P}<0.01$; $* * *, p<0.001$.

\section{MAGI2-AS3 inactivated STAT signaling in prostate cancer cells}

To explore how MAGI2-AS3 regulated cell apoptosis, we detected expression of Bcl-2 and Mcl-1, two key regulators of cell apoptosis, in cells transfected with MAGI2-AS3. MAGI2-AS3 overexpression decreased Bcl-2 and Mcl-1 mRNA in PC-3 and DU145 cells (Fig. 3A-B). Bcl-2 and Mcl-1 were important targets of STAT signaling in prostate cancer. We transfected STAT3 reporter vector into PC-3 cells. As a positive control, IL6 activated STAT3 reporter (Fig. 3C). On the contrary, MAGI2-AS3 overexpression decreased activity of STAT3 reporter, which was reversed by IL6 administration (Fig. 3C). Furthermore, western blotting showed that MAGI2-AS3 overexpression decreased both p-STAT3 and total STAT3 protein expression in PC-3 and DU145 cells (Fig. 3D).

\section{MAGI2-AS3 and miR-424-5p mutually repressed each other in prostate cancer cells}

Via bioinformatic analysis, we found a binding site for miR-424-5p in the sequence of MAGI2-AS3 (Fig. 4A). In PC-3 and DU145 cells, MAGI2-AS3 overexpression decreased miR-424-5p levels (Fig. 4B). We also transfected miR-424-5p mimic into PC-3 and
DU145 cells to upregulate miR-424-5p expression (Fig. $4 C)$. Elevation of miR-424-5p decreased MAGI2-AS3 expression in PC-3 and DU145 (Fig. 4D). To confirm their direct interaction, MAGI2-AS3 and the mutation form with two-point mutations were inserted into luciferase reporter vector (Fig. 4E). In PC-3 cells, miR-424-5p mimic repressed luciferase activity in cells transfected with MAGI2-AS3 not MAGI2-AS3-Mut (Fig. 4F). Consistently, similar results were observed in DU145 cells (Fig. 4G). More importantly, RIP assay suggested that MAGI2-AS3 and miR-424-5p were both enriched in AGO2 antibody (Fig. 4H), indicating their direct interaction.

\section{MAGI2-AS3 regulated COP1 to repress STAT3 activity in prostate cancer cells}

miR-424-5p regulated STAT3 via targeting COP1 [11]. We found that COP1 mRNA levels were increased by MAGI2-AS3, while decreased by miR-424-5p mimic, in addition, miR-424-5p overexpression reversed the effect of MAGI2-AS3 on COP1 expression (Fig. 5A). Similarly, western blotting also showed that MAGI2-AS3 increased COP1 protein expression, meanwhile, miR-424-5p decreased COP1 protein expression and reversed the effect of MAGI2-AS3 on COP1 protein level (Fig. 5B). 
Moreover, miR-424-5p mimic elevated $\mathrm{Bcl}-2$ and Mcl-1 mRNA levels and reversed the downregulation of Bcl-2 and Mcl-1 mRNA levels induced by MAGI2-AS3 in PC-3 and DU145 cells (Fig. 5C-D).
Additionally, miR-424-5p could revere the elevation of STAT3 activity by MAGI2-AS3 overexpression (Fig. $5 \mathrm{E})$.
A

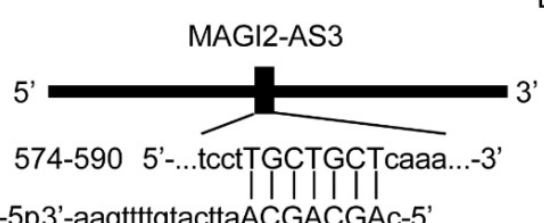

miR-424-5p3'-aagtttgtacttaACGACGAc-5'

C

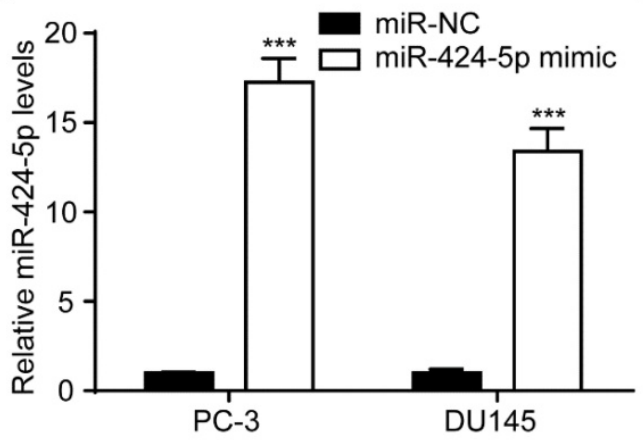

E

MAGI2-AS3-WT 5'-...tcctTGCTGCTcaaa...-3' miR-424-5p 3'-aagttttgtacttaACGACGAC-5 (IX I| MAGI2-AS3-Mut 5'-...tcctTGGGGCTcaaa...-3'

G

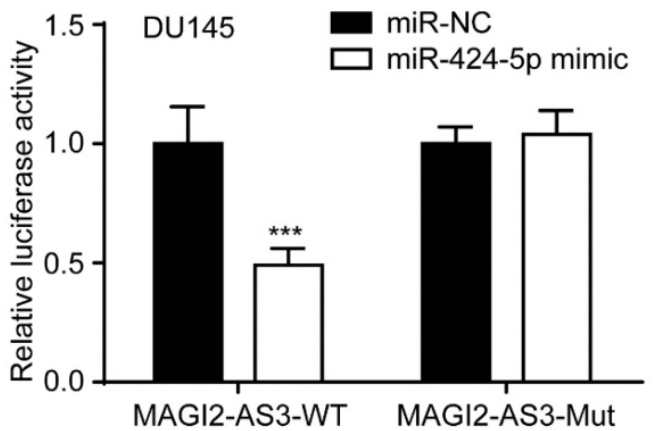

D

$\mathrm{F}$

$\mathrm{H}$
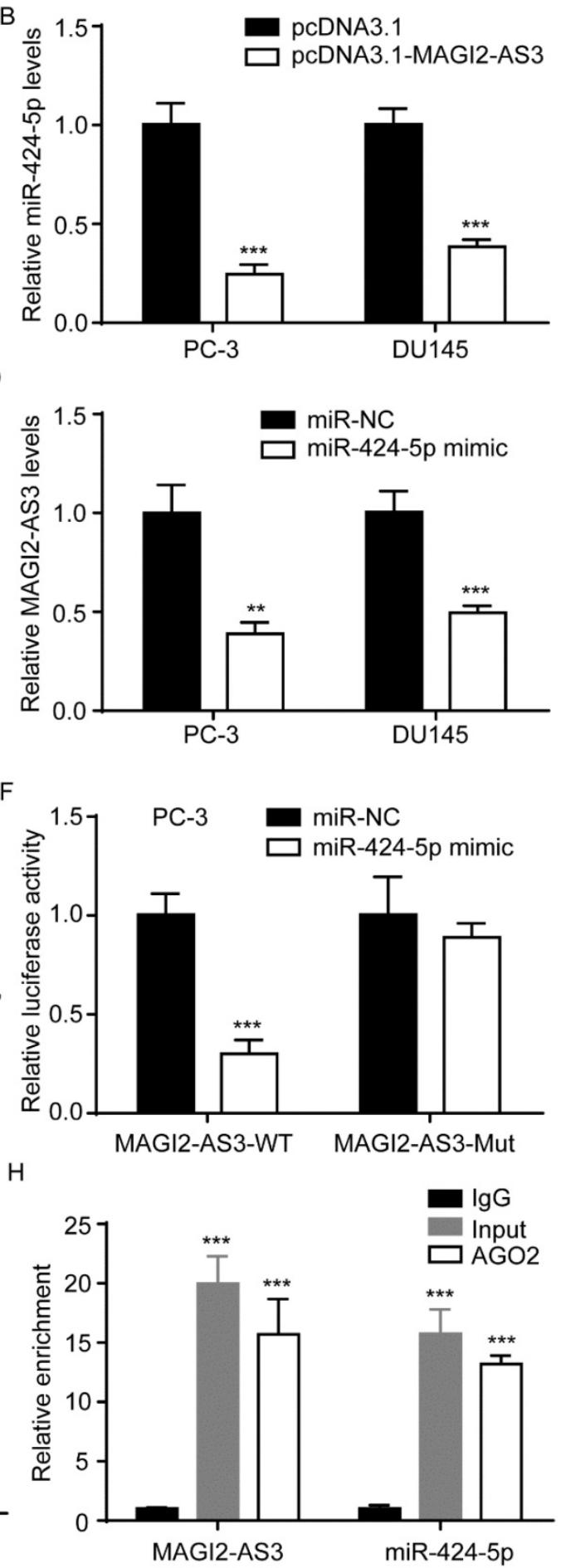

Figure 4. MAGI2-AS3 regulated miR-424-5p expression in prostate cancer cells. A. A scheme of binding site for miR-424-5p in $M A G I 2-A S 3$ sequence. B. miR-424-5p expression was detected in PC-3 and DU145 cells with transfection of pcDNA3.1 or pcDNA3.1-MAGI2-AS3. C. miR-424-5p expression was detected in PC-3 and DU145 cells with transfection of miR-NC or miR-424-5p mimic. D. MAGI2-AS3 expression was detected in PC-3 and DU145 cells with transfection of miR-NC or miR-424-5p mimic. E. Sequences of MAGI2-AS3-WT and MAGI2-AS3-Mut. F-G. The relative luciferase activity was determined in PC-3 (F) and DU145 (G) cells with transfection of miR-NC or miR-424-5p mimic. H. RIP was performed to detect enrichment of MAGI2-AS3 and miR-424-5p on AGO2. $* *, p<0.01 ; * * *, p<0.001$. 
A

control

MAGI2-AS3

$\exists$ miR-424-5p mimic

MAGI2-AS3+miR-424-5p mimic

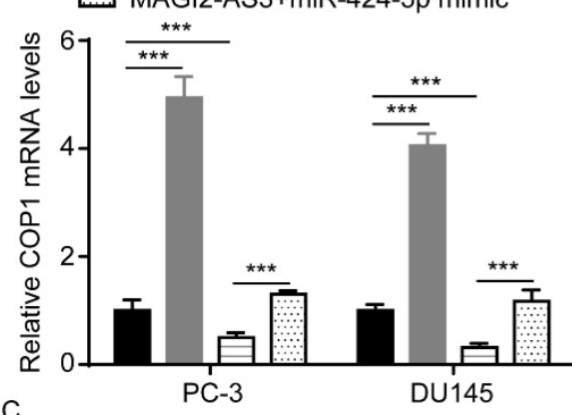

C

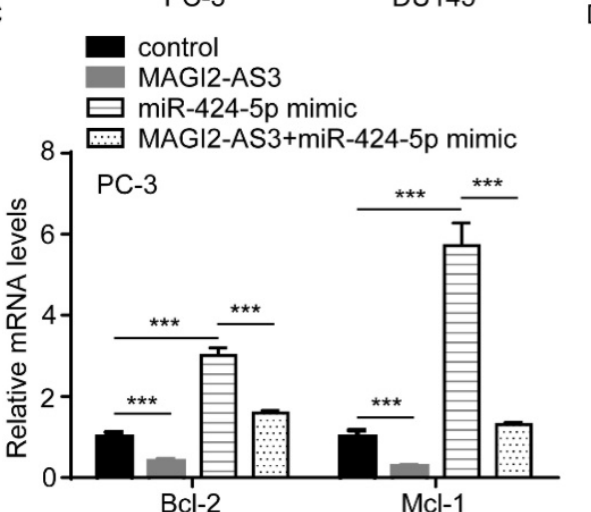

E

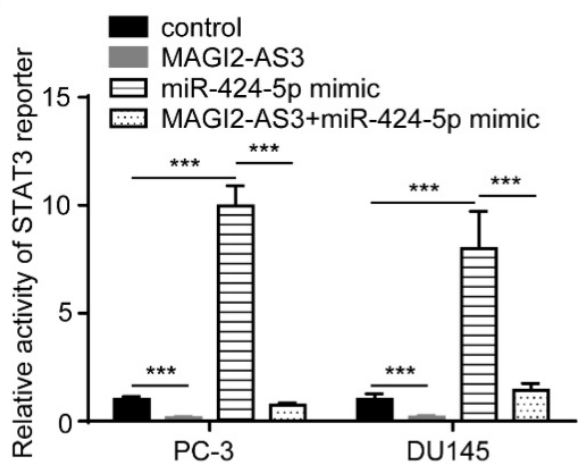

B
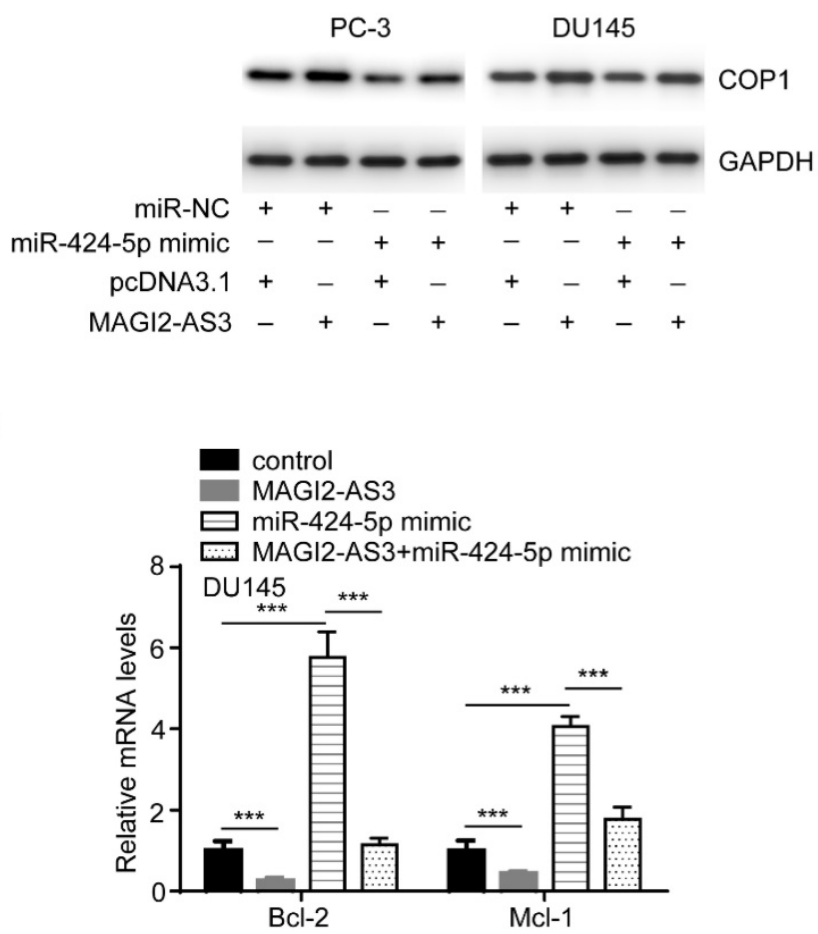

Figure 5. MAGI2-AS3 inactivated STAT3 signaling by sponging miR-424-5p. A-B. COPI mRNA (A) and protein (B) levels were detected in PC-3 and DUI 45 cells with transfection of pcDNA3.1 or pcDNA3.1-MAGI2-AS3 and miR-NC or miR-424-5p mimic. C-D. Bcl-2 and Mcl-1 mRNA levels were detected in PC-3 (C) and DU145 (D) cells with transfection of pcDNA3.1 or pCDNA3.1-MAGI2-AS3 and miR-NC or miR-424-5p mimic. E. STAT3 reporter activity was detected in PC-3 and DU145 cells with transfection of pcDNA3.1 or pcDNA3.1-MAGI2-AS3 and miR-NC or miR-424-5p mimic. ***, $\mathrm{p}<0.001$.

\section{MAGI2-AS3 regulated prostate cancer cell proliferation via inactivation of STAT3 signaling}

To study whether STAT3 signaling was pivotal for the function of MAGI2-AS3, we combined IL6 treatment with recombinant MAGI2-AS3 in PC-3 and DU145 cells. As we expected, IL6 administration attenuated the effect of MAGI2-AS3 overexpression on cell proliferation and apoptosis (Fig. 6A-D).

\section{MAGI2-AS3 was correlated with miR-424-5p, COP1, Bcl-2 and Mcl-1 expression in clinical samples}

RT-qPCR showed that miR-424-5p levels were upregulated in collected prostate tumors and its expression was negatively correlated with MAGI2-AS3 expression (Fig. 7A-B). We further found that COP1 mRNA levels were downregulated in collected prostate tumors, which was positively correlated with MAGI2-AS3 expression (Fig. 7C-D). In TCGA-PRAD dataset, it was revealed that MAGI2-AS3 expression was positively correlated with COP1 mRNA levels (Fig. 7E). In conclusion, the current data suggested that MAGI2-AS3 acted as a sponge for miR-424-5p to elevate COP1 expression and inactivated STAT3 signaling in prostate cancer cells (Fig. 7F). 


\section{Discussion}

In recent years, a large number of aberrant expressed lncRNAs have been found to be associated with initiation, uncontrolled cell proliferation, metastasis and drug resistance of cancer [12]. In particular, researchers found that several lncRNAs could mediate resistance to cell death to maintain cell proliferation in prostate cancer cells $[13,14]$. According to WGCNA analysis of datasets (TCGA, GSE17951, GSE7076), Cai et al. found that MAGI-AS3 was one of the downregulated lncRNAs in prostate cancer and was one of prognosis-related genes for patients with prostate cancer [10]. In the present study, we also retrieved TCGA and GEO data (GSE46602), consistently, significant downregulation of MAGI2-AS3 was found. Moreover, we collected patient samples for validation. In consistent with the microarray and RNA sequencing data, RT-qPCR also showed that MAGI2-AS3 expression was decreased in prostate cancer. However, the role of MAGI2-AS3 in cancer was controversial. In non-small cell lung cancer, breast cancer and esophageal cancer, MAGI2-AS3 played a key role in inhibiting cell proliferation and serving as a radiotherapy sensitizer [15-17]; on the contrary, MAGI-AS3 functioned as an oncogenic potential in gastric cancer and colorectal cancer $[9,18]$. Herein, we found that in prostate cancer cells, forced elevation of MAGI2-AS3 inhibited prostate cancer cell proliferation and induced cell apoptosis, supporting a tumor suppressor role of MAGI2-AS3 in prostate cancer.

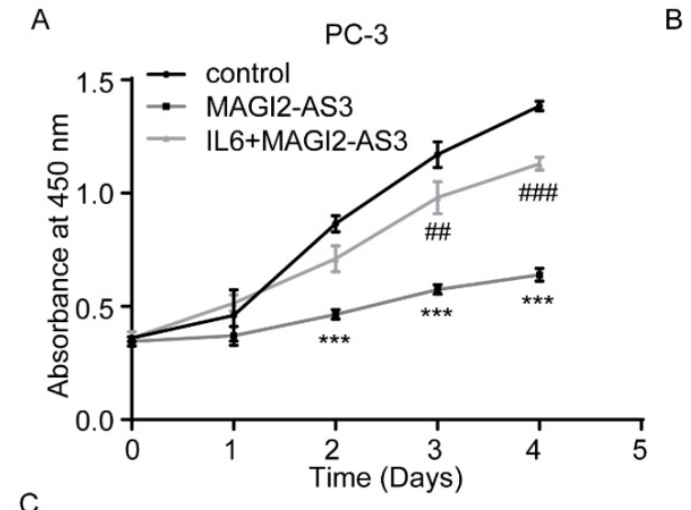

B
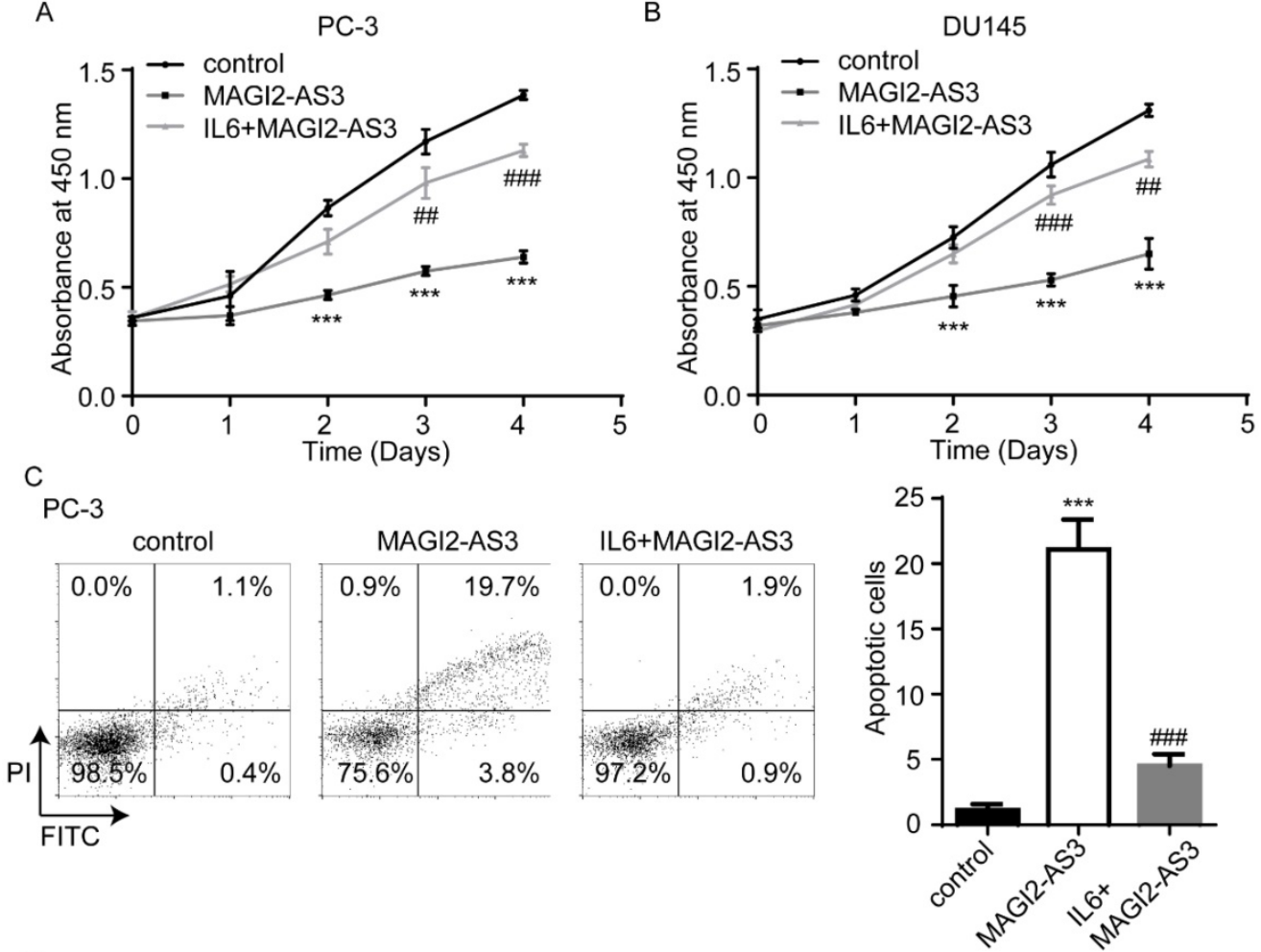

D DU145
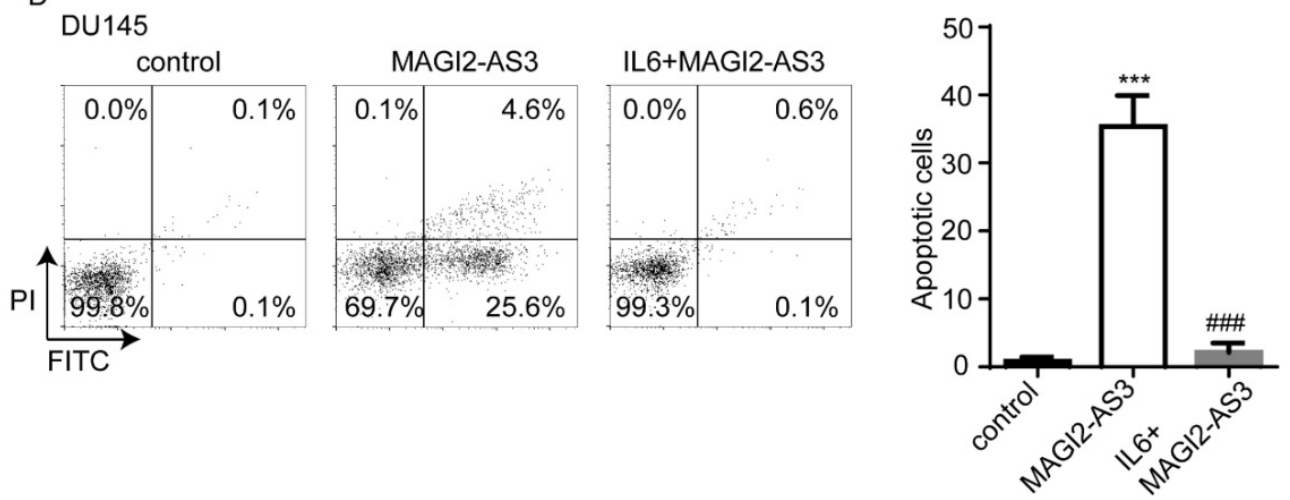

Figure 6. MAGI2-AS3 regulated prostate cancer cell proliferation via controlling STAT3 activity. A-B. The cell proliferation ability was detected in PC-3 (A) and DU145 (B) cells with transfection of pcDNA3.1 or pcDNA3.1-MAGI2-AS3 and treatment of IL6. C-D. The percentage of apoptotic cells was detected in PC-3 (C) and DU145 (D) cells with transfection of pcDNA3.1 or pcDNA3.1-MAGI2-AS3 and treatment of IL6. *** vs. control group, $\mathrm{p}<0.001$; \#\# vs. MAGI2-AS3 group, $\mathrm{p}<0.001$. 
A

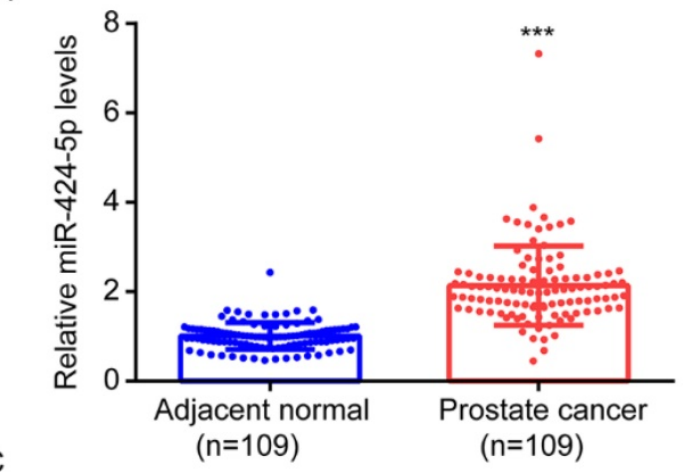

C

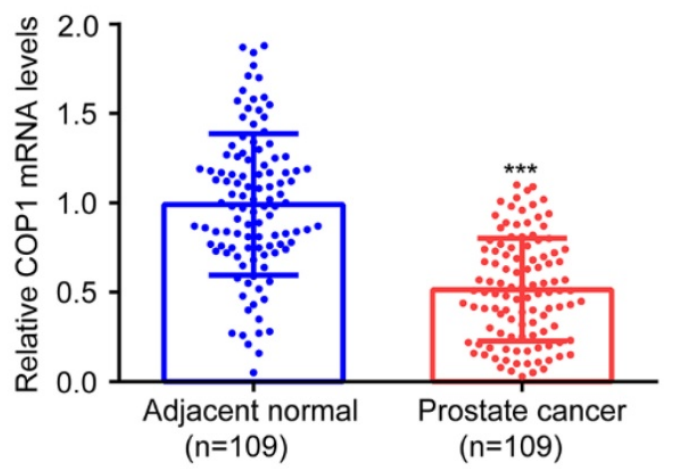

E

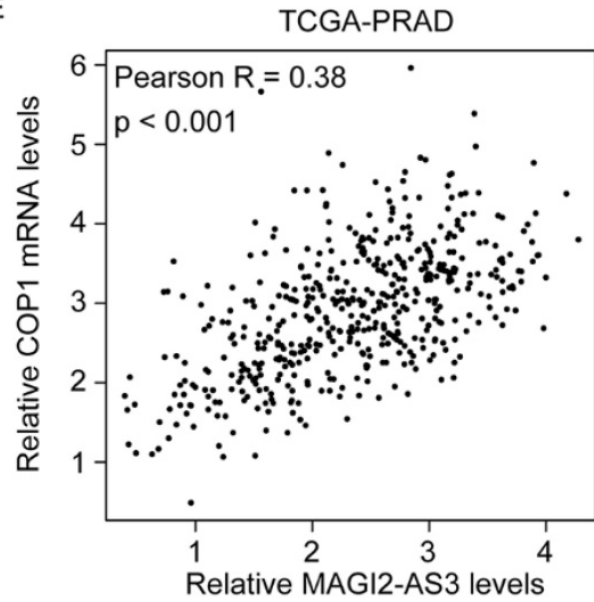

$\mathrm{F}$
B

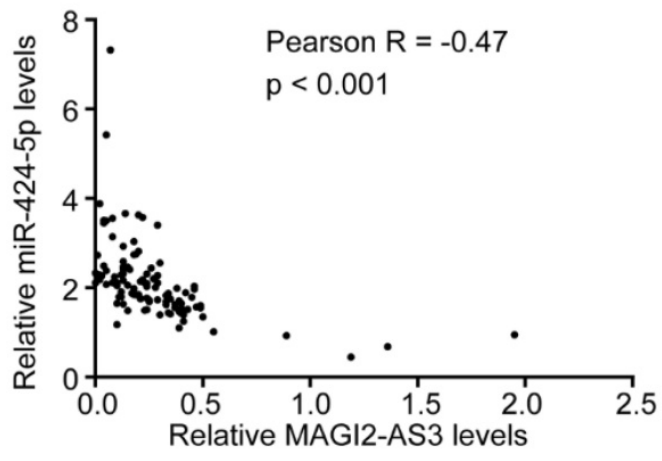

D

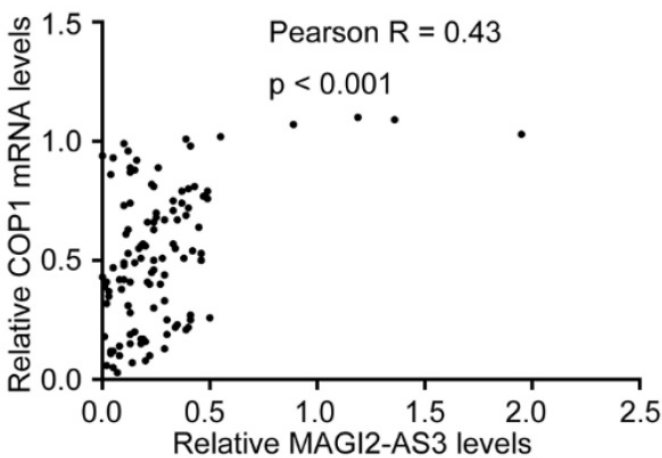

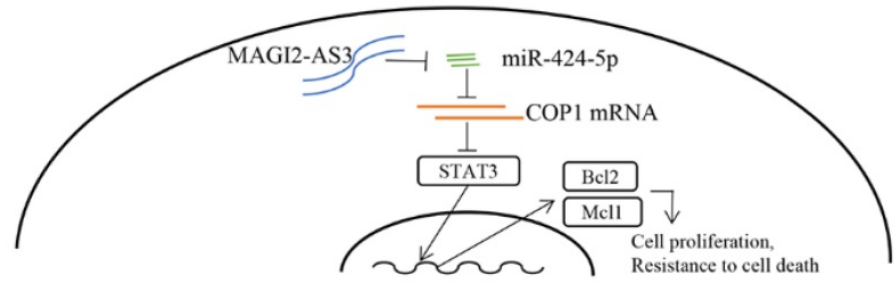

Figure 7. MAGI2-AS3 expression was associated with miR-424-5p and COP 1 expression in clinical samples. A. miR-424-5p expression was detected in 109 pairs of prostate cancer and adjacent normal prostate by RT-qPCR. B. The association between MAGI2-AS3 and miR-424-5p levels was analyzed in collected samples by Pearson correlation analysis. C. COP1 mRNA expression was detected in 109 pairs of prostate cancer and adjacent normal prostate by RT-qPCR. D. The associated between MAGI2-AS3 and COP1 mRNA levels was analyzed in collected samples by Pearson correlation analysis. E. The association between MAGI2-AS3 and COP1 mRNA levels was analyzed in TCGA data by Pearson correlation analysis. F. A model of the MAGI2-AS3/miR-424-5p/COPI axis in prostate cancer cells. ***, $p<0.001$.

STAT3 signaling was one of the well-characterized cancer driver pathways in prostate cancer, which was critical for maintaining uncontrolled cell proliferation [19]. The activity of STAT3 signaling was tightly controlled by its interactors and several of them were excellent drug targets [20]. The hyper-activity of STAT3 signaling was partially due to the dysregulation of non-coding RNAs including lncRNAs in prostate cancer cells [21, 22]. The activated STAT3 upregulated Bcl-2, Mcl-1 and several other apoptosis-related genes to mediate resistance to cell apoptosis [23]. As we showed in our findings, MAGI2-AS3 promoted prostate cancer cell apoptosis, and repressed the activity of STAT3 signaling. The findings added new sights into understanding the regulation of STAT3 signaling in prostate cancer.

Many lncRNAs exerted their function via acting as miRNA sponges. Mainly localized in cytoplasm, MAGI2-AS3 also sponged several miRNAs to regulate cancer progression. It was revealed that MAGI2-AS3 could sponge miR-141/200a, miR-25 and miR-3163 in different cell backgrounds $[9,16,18]$. Similar to MAGI2-AS3, miR-424-5p could act as an oncogene or 
tumor suppressor in different cancer types. miR-424-5p facilitated cancer progression of prostate cancer and inhibited hepatocellular carcinoma development [11, 24]. Interestingly, we found a binding site for miR-424-5p in the middle area of $M A G I 2-A S 3$ sequence, we further confirmed that MAGI2-AS3 and miR-424-5p mutually repressed the expression of each other. Their direct interaction was confirmed by dual luciferase activity assay and RIP assays. miR-424-5p targeted E3 ligase COP1 to stabilize STAT3, thus promoting cell proliferation of prostate cancer cells [11]. Our data also showed that COP1 was upregulated and STAT3 was downregulated in prostate cancer cells with overexpression of MAGI2-AS3, moreover, the effects of MAGI2-AS3 on COP1 was reversed by miR-424-5p mimic. These results indicated that MAGI2-AS3 activated STAT3 signaling via sponge miR-424-5p in prostate cancer cells.

Collectively, the current study defined MAGI2-AS3 as a novel tumor suppressor in prostate cancer and suggested MAGI2-AS3 as a biomarker and drug target for the patients with prostate cancer.

\section{Acknowledgements}

\section{Ethical statement}

Informed consent was collected from all participants. The protocol of the current study was approved by the Ethical Committee of China-Japan Union Hospital of Jilin University (IRB number: 2016-21).

\section{Funding}

The research did not receive any specific grant from funding agencies in the public, commercial, or not-for-profit sectors.

\section{Competing Interests}

The authors have declared that no competing interest exists.

\section{References}

1. Siegel RL, Miller KD and Jemal A. Cancer statistics, 2018. CA: A Cancer Journal for Clinicians. 2018; 68: 7-30.

2. Du Z, Sun T, Hacisuleyman E, Fei T, Wang X, Brown M, et al. Integrative analyses reveal a long noncoding RNA-mediated sponge regulatory network in prostate cancer. Nat Commun, 2016; 7: 10982.

3. Lin JZ, Wang WW, Hu TT, Zhu GY, Li LN, Zhang CY, et al. FOXM1 contributes to docetaxel resistance in castration-resistant prostate cancer by inducing AMPK/mTOR-mediated autophagy. Cancer Lett, 2020; 28: 481-489.

4. Yu Y, Gao F, He Q, Li G, Ding G. IncRNA UCA1 Functions as a ceRNA to Promote Prostate Cancer Progression via Sponging miR143. Mol Ther Nucleic Acids, 2020; 19: 751-758.

5. Wu M, Huang Y, Chen T, Wang W, Yang S, Ye Z, et al. LncRNA MEG3 inhibits the progression of prostate cancer by modulating miR-9-5p/QKI-5 axis. J Cell Mol Med, 2019; 23: 29-38.

6. Hua JT, Chen S, He HH. Landscape of Noncoding RNA in Prostate Cancer. Trends Genet, 2019; 35: 840-851.

7. Tian T, Gong Z, Wang M, Hao R, Lin S, Liu K, et al. Identification of long non-coding RNA signatures in triple-negative breast cancer. Cancer Cell Int, 2018; 18: 103 .
8. Zhu N, Hou J, Wu Y, Liu J, Li G, Zhao W, et al. Integrated analysis of a competing endogenous RNA network reveals key lncRNAs as potential prognostic biomarkers for human bladder cancer. Medicine (Baltimore), 2018; 97: e11887.

9. Li D, Wang J, Zhang M, Hu X, She J, Qiu X, et al. LncRNA MAGI2-AS3 Is Regulated by BRD4 and Promotes Gastric Cancer Progression via Maintaining ZEB1 Overexpression by Sponging miR-141/200a. Mol Ther Nucleic Acids, 2020; 19: 109-123.

10. Cai J, Chen Z, Chen X, Huang H, Lin X, Miao B. Coexpression Network Analysis Identifies a Novel Nine-RNA Signature to Improve Prognostic Prediction for Prostate Cancer Patients. Biomed Res Int, 2020; 1: 4264291.

11. Dallavalle C, Albino D, Civenni G, Merulla J, Ostano P, Mello-Grand M, et al. MicroRNA-424 impairs ubiquitination to activate STAT3 and promote prostate tumor progression. J Clin Invest, 2016; 126: 4585-4602.

12. Wei L, Wang X, Lv L, Zheng Y, Zhang N, Yang M. The emerging role of noncoding RNAs in colorectal cancer chemoresistance. Cell Oncol, 2019; 42: 757-768.

13. Misawa A, Takayama K, Urano T, Inoue S. Androgen-induced Long Noncoding RNA (lncRNA) SOCS2-AS1 Promotes Cell Growth and Inhibits Apoptosis in Prostate Cancer Cells. J Biol Chem, 2016; 291: 17861-80.

14. Zhu Y, Tong Y, Wu J, Liu Y, Zhao M. Knockdown of LncRNA GHET1 suppresses prostate cancer cell proliferation by inhibiting HIF-1a/Notch-1 signaling pathway via KLF2. Biofactors, 2019; 45: 364-373.

15. Xu X, Yuan X, Ni J, Guo J, Gao Y, Yin W, et al. MAGI2-AS3 inhibits breast cancer by downregulating DNA methylation of MAGI2. J Cell Physiol, 2021; 236: 1116-1130.

16. Sui $\mathrm{Y}$, Chi W, Feng L, Jiang J. LncRNA MAGI2-AS3 is downregulated in non-small cell lung cancer and may be a sponge of miR-25. BMC Pulm Med, 2020; 20: 59 .

17. Cheng $\mathrm{W}$, Shi $\mathrm{X}$, Lin $\mathrm{M}$, Yao $\mathrm{Q}$, Ma J, Li J. LncRNA MAGI2-AS3 Overexpression Sensitizes Esophageal Cancer Cells to Irradiation Through Down-Regulation of HOXB7 via EZH2. Front Cell Dev Biol, 2020; 8: 552822.

18. Ren H, Li Z, Tang Z, Li J, Lang X. Long noncoding MAGI2-AS3 promotes colorectal cancer progression through regulating miR-3163/TMEM106B axis. J Cell Physiol, 2020; 235: 4824-4833.

19. Carpenter RL, Lo HW. STAT3 Target Genes Relevant to Human Cancers. Cancers (Basel), 2014; 6: 897-925.

20. Laudisi F, Cherubini F, Monteleone G, Stolfi C. STAT3 Interactors as Potential Therapeutic Targets for Cancer Treatment. Int J Mol Sci, 2018; 19: 1787.

21. Luo J, Wang K, Yeh S, Sun Y, Liang L, Xiao Y, et al. LncRNA-p21 alters the antiandrogen enzalutamide-induced prostate cancer neuroendocrine differentiation via modulating the EZH2/STAT3 signaling. Nat Commun, 2019; 10: 2571

22. Wang N, Jiang $\mathrm{Y}, \mathrm{Lv} \mathrm{S}$, Wen $\mathrm{H}, \mathrm{Wu} \mathrm{D}$, Wei Q, et al. HOTAIR expands the population of prostatic cancer stem-like cells and causes Docetaxel resistance via activating STAT3 signaling. Aging (Albany NY), 2020; 12: 12771-12782.

23. $\mathrm{Hu} \mathrm{H}$, Lee $\mathrm{HJ}$, Jiang $\mathrm{C}$, Zhang J, Wang $\mathrm{L}$, Zhao $\mathrm{Y}$, et al. Penta-1,2,3,4,6-O-galloyl-beta-D-glucose induces p53 and inhibits STAT3 in prostate cancer cells in vitro and suppresses prostate xenograft tumor growth in vivo. Mol Cancer Ther, 2008; 7: 2681-91.

24. Teng F, Zhang JX, Chang QM, Wu XB, Tang WG, Wang JF, et al. LncRNA MYLK-AS1 facilitates tumor progression and angiogenesis by targeting miR-424-5p/E2F7 axis and activating VEGFR-2 signaling pathway in hepatocellular carcinoma. J Exp Clin Cancer Res, 2020; 39: 235. 